\title{
A Perfect Metamaterial Absorber
}

\author{
N. I. Landy \\ Boston College, Department of Physics, 140 Commonwealth Ave., Chestnut Hill, MA 02467. \\ S. Sajuyigbe, J. J. Mock, D. R. Smith \\ Department of Electrical and Computer Engineering, Duke University, Durham, NC 27708 USA. \\ W. J. Padilla \\ Boston College, Department of Physics, 140 Commonwealth Ave., Chestnut Hill, MA 02467.
}

(Dated: March 13, 2008)

\begin{abstract}
We present the design for an absorbing metamaterial element with near unity absorbance. Our structure consists of two metamaterial resonators that couple separately to electric and magnetic fields so as to absorb all incident radiation within a single unit cell layer. We fabricate, characterize, and analyze a metamaterial absorber with a slightly lower predicted absorbance of $96 \%$. This achieves a simulated full width at half maximum (FWHM) absorbance of $4 \%$ thus making this material ideal for imaging purposes. Unlike conventional absorbers, our metamaterial consists solely of metallic elements. The underlying substrate can therefore be chosen independently of the substrate's absorptive qualities and optimized for other parameters of interest. We detail the design and simulation process that led to our metamaterial, and our experiments demonstrate a peak absorbance greater than $88 \%$ at $11.5 \mathrm{GHz}$.
\end{abstract}

The nascent field of electromagnetic metamaterials has produced exotic effects such as negative index of refraction [1, 2], and devices such as an electromagnetic cloak 3 . The realization of such properties lies in the ability of metamaterials to create independent tailored electric [4] and magnetic [5] responses to incident radiation. Electromagnetic metamaterials are also geometrically scalable which translates into operability over a significant portion of the electromagnetic spectrum. To date metamaterials have been demonstrated in every technologically relevant spectral range, from radio 6], microwave 7, mm-Wave 8, THz 9, MIR [10, NIR [1], to the near optical 12. These designer electromagnetic materials are an ideal platform for the investigation of novel emergent physical phenomena while also holding great promise for future applications.

As an effective medium [13, metamaterials can be characterized by a complex electric permittivity $\tilde{\epsilon}(\omega)=$ $\epsilon_{1}+i \epsilon_{2}$ and magnetic permeability $\tilde{\mu}(\omega)=\mu_{1}+i \mu_{2}$. Much of the work in metamaterials has been focussed on the real part of $\epsilon$ and $\mu$ to enable the creation of a negative refractive material. However, the oft-overlooked loss components of the optical constants $\left(\epsilon_{2}\right.$ and $\left.\mu_{2}\right)$ have much potential for the creation of exotic and useful materials as well. For example, they can be manipulated to create a high absorber. By manipulating electric and magnetic resonances independently, it is possible to absorb both the incident electric and magnetic field. Additionally, by matching $\epsilon$ and $\mu$, a metamaterial can be impedance-matched to free space, minimizing reflectivity. In this paper, we show that metamaterials can be fashioned to create narrow-band perfect absorbers, with the potential to be used in devices such as in bolometers.

A single unit cell of the absorber consisted of two dis- (a)

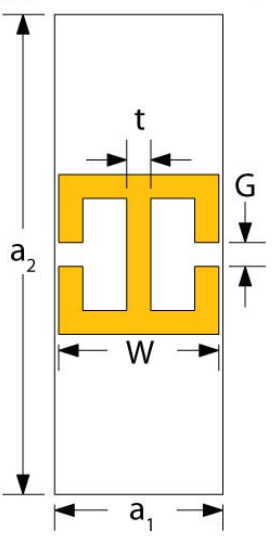

(b)

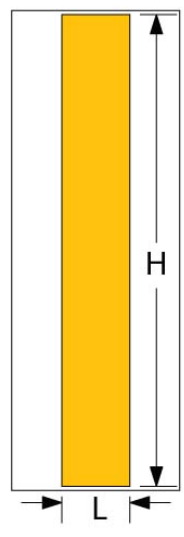

(c)

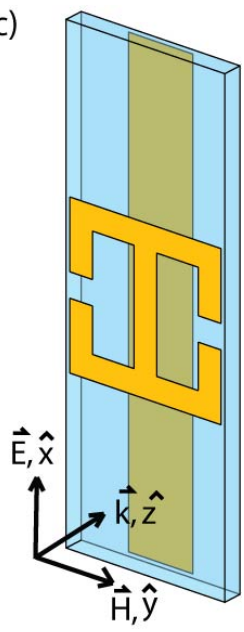

FIG. 1: Electric resonator (a) and cut wire (b). Dimension notations are listed in (a) and (b). The unit cell is shown in (c) with axes indicating the direction of propagation of a TEM wave.

tinct metallic elements as shown in Fig. 1 (a) and (b). Electric coupling was supplied by the electric ring resonator (ERR), similar to the design presented by Padilla et al. 14, and is shown in Fig. 1] (a). This element consisted of two standard split ring resonators connected by the inductive ring parallel to the split-wire. We used this design instead of a conventional split-wire design because of the limitations of straight wire media [15, i.e. a splitwire design has limited tunability beyond the addition of more wires per unit cell to increase inductance [16]. The magnetic coupling required a more complicated arrangement, and thus in order to couple to the incident $\mathbf{H}$-field, 


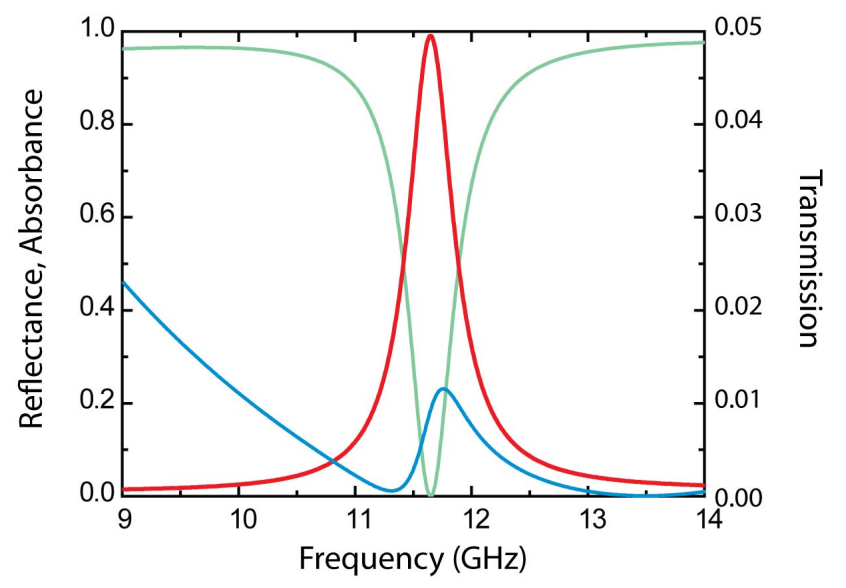

FIG. 2: Simulated perfect metamaterial absorber. Reflectance (green curve) and absorbance (red curve) are plotted from zero to $100 \%$ (left axis). The transmission is plotted on the right axis as the blue curve on a scale from zero to $5 \%$.

we needed flux created by circulating charges perpendicular to the propagation vector. We created this response by combining the center wire of the electric resonator with a cut wire (Fig. 11(b)) in a parallel plane separated by a substrate (Fig. 1(c)). This design is similar to the so-called "fishnet" and paired nanorod structures [11, 12, in the sense that they derive a magnetic response from driving two antiparallel currents in conducting segments. We were then able to tune the magnetic response by changing the geometry of the cut wire and the separation between the cut wire and electric resonator. By manipulating the magnetic coupling without changing the geometry of the electric resonator, we able to decouple $\epsilon$ and $\mu$, and therefore individually tune each resonance.

We performed computer simulations of an ideal - but realizable - metamaterial absorber using the commercial finite-difference time domain (FDTD) solver Microwave Studio by CST. 17 The program simulated a single unit cell as shown in Fig. 11 (c) with appropriate boundary conditions, i.e. perfect electric ( $\hat{y} \hat{z}$-plane) and perfect magnetic ( $\hat{x} \hat{z}$-plane). Waveguide ports on the other boundaries simulated a TEM plane wave propagating through the medium. Simulation produced the complex frequency dependent S-parameters, $\widetilde{S}_{11}$ and $\widetilde{S}_{21}$, where $T(\omega)=\left|S_{21}\right|^{2}$ and $R(\omega)=\left|S_{11}\right|^{2}$ are the transmission and reflectance, respectively. From the S-parameter data we inverted the fresnel equations to extract the complex optical constants [18. We then examined the behavior of the surface current density, magnetic and electric fields at $\omega_{0}$ to verify that we were coupling to the correct resonant mode of each metamaterial element. By tuning $\omega_{0}$ of the $\epsilon$ and $\mu$ resonances (not shown), due to the two metamaterial elements, we achieved $\epsilon=\mu$ and thus an impedance near the free space value. While $100 \%$ asborbance is theoretically possible, it can only occur when the metamaterial layer is impedance-matched to free space such that the reflectance is zero. [19] One may then minimize transmission such that the addition of multiple layers ensures $T(\omega) \rightarrow 0$. The simulated metamaterial had the dimensions, in millimeters, of: $\mathrm{a}_{1}=4.2$, $\mathrm{a}_{2}=12, \mathrm{~W}=3.9, \mathrm{G}=0.606, \mathrm{t}=0.6, \mathrm{~L}=1.7, \mathrm{H}=11.8$, and the metamaterials elements were separated by 0.65 in the $\hat{z}$ direction. In Fig. 2 we show the simulation results for the metamaterial perfect absorber. Reflectance and absorbance are plotted from zero to $100 \%$ (left axis) and the transmission is plotted on the right axis from zero to $5 \%$. The reflectance is large $\sim 97 \%$ near the bounds of the plot, $9 \mathrm{GHz}$ and $14 \mathrm{GHz}$, but there is a minimum of $0.01 \%$ at $\omega_{0} \equiv 11.65 \mathrm{GHz}$. The simulated transmission also undergoes a minimum near $\omega_{0}$ and yields a value of $\sim 0.9 \%$. Thus we achieve a best simulated absorbance $A(\omega)=1-T(\omega)-R(\omega)$ slightly less than unity $A(\omega)=99 \%$ with a FWHM of $4 \%$ compared to $\omega_{0}$.

While our simulated absorber achieves narrow-band high absorbance, we were restricted by the minimum line widths available to us $(250 \mu \mathrm{m})$ and other various fabrication tolerances. These limitations were incorporated into the design process and we fabricated a metamaterial which deviated slightly from the ideal absorber and had the dimensions, in millimeters, of: $\mathrm{a}_{1}=4.2, \mathrm{a}_{2}=12$, $\mathrm{W}=4, \mathrm{G}=0.6, \mathrm{t}=0.6, \mathrm{~L}=1.7, \mathrm{H}=11.8$. Each metallization was fabricated on a FR4 substrate with a thickness of $0.2 \mathrm{~mm}$. Metamaterials were fabricated using standard optical lithography. Photosensitized half-ounce copperclad FR-4 circuit board formed the substrate material with an $17 \mu \mathrm{m}$ copper thickness. A mask was designed and printed in high resolution on a transparency. The photosensitized copper clad FR-4 was covered by the mask and exposed to ultraviolet light then developed in a sodium carbonate solution and etched in ferric chloride. Finally, the board was placed in a stripper solution to remove the remaining photoresist. Both the ERR layer (Fig. 1(a)) and the cut-wire layer (Fig. 1(b)) were fabricated in such a fashion, see inset to Fig. 3 (b). These boards where then sandwiched (using an adhesive with $0.06 \mathrm{~mm}$ thickness) about another $0.2 \mathrm{~mm}$ thick FR4 blank substrate to obtain the correct spacing. The end results was that the metamaterials elements were separated by $0.72 \mathrm{~mm}$ in the $\hat{z}$ direction. This design allowed us to couple to both magnetic and electric resonances without using out-of-plane elements typical with "winecrate" designs, [2] thus greatly simplifying construction of the absorber.

We experimentally verified the behavior of the absorber by measuring the complex S-parameters of a large planar array of pixels (outer dimensions of $15 \times 15 \mathrm{~cm}$ ). We used an Agilent vector network analyzer that produced microwaves in the range of 8-12 GHz. One microwave horn (Rozendal and Associates) focused the $\mathrm{GHz}$ 


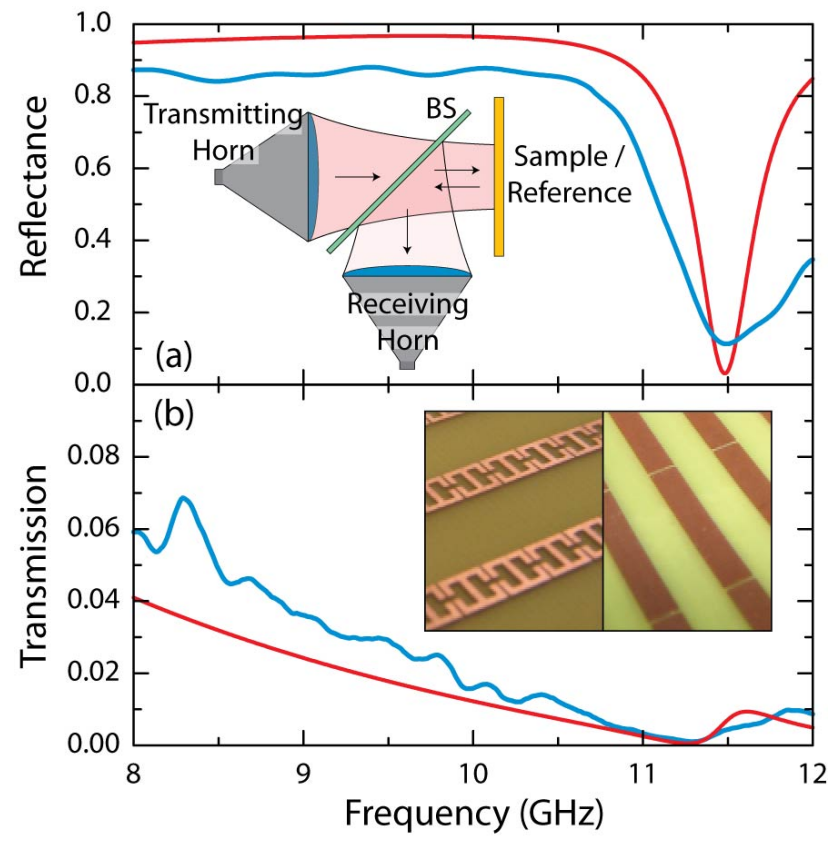

FIG. 3: Simulated (red curves) and measured (blue curves) $\mathrm{T}(\omega)$ and $\mathrm{R}(\omega)$ for the microwave absorber. (a) displays the simulated and measured reflectance from zero to $100 \%$. A schematic of the reflection experiment is shown as the inset to (a) where BS is the beam splitter. The simulated and measured transmission, shown in (b), are displayed from zero to $10 \%$. Both reach a minimum of approximately $0.6 \%$ near $11.5 \mathrm{GHz}$. Inset to (b) shows photographs of the individual components which make up the metamaterial absorber. The sample consists of the electric ring resonator (left) and the split wire (right) and is joined with an FR4 spacer of $0.72 \mathrm{~mm}$ thickness.

beam on the sample and another horn served as a detector. Both horns coupled to linearly polarized light and had parallel polarization directions. To measure transmission, we set the horns in a normal incidence confocal configuration. We performed reflectance measurements at normal incidence. However, in order to eliminate large voltage standing wave ratio (VSWR) which would overwhelm the data, we used a beam splitter configuration, shown as the inset to Fig. 3 (a). Radiation first traveled from the transmitting horn through the beam splitter, reflected from the sample and returned to the beam splitter where it was reflected at $45 \%$ to the receiving horn. We set the normalization and phase in the transmission configuration by simply removing the sample. For reflection, we replaced the sample with a perfect reflector for normalized measurements. Transmission characterization techniques utilized here have been described elsewhere in detail[20.

Computer simulations were performed for this metamaterial structure and are presented for comparison to experimental data. The simulated $R(\omega)$ is shown as the

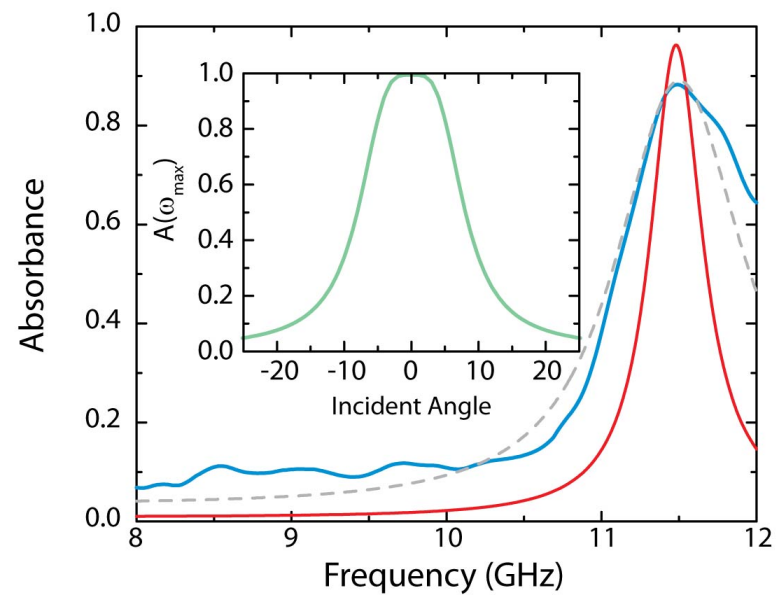

FIG. 4: Main panel shows the simulated (red) and measured (blue) absorbance curves. Significant broadening in the reflection curve contributes to the deviation of the experimental absorbance curve from simulation. The dashed gray absorbance curve is a Gaussian weighted average which approximates assembly error of the metamaterials absorber, described in the text. Inset shows the simulated angular dependence of the absorbance at at $\omega_{\max }$.

red curve in Fig. 3 (a). At low frequencies the reflectivity is high and yields values near 95\%. A large feature occurs in $R(\omega)$ and has a minimum of $\sim 3 \%$ near $11.5 \mathrm{GHz}$. Likewise the simulated transmissivity, shown as the red curve in Fig. 3 (b), has $4 \% T(\omega)$ at low frequencies and then undergoes a minimum near $11.25 \mathrm{GHz}$. The simulated absorbance of our metamaterial unit cell thus has a maximum near where both $R(\omega)$ and $T(\omega)$ have their minima. We plot $A(\omega)$ as the red curve in Fig. 4 from 8 to $12 \mathrm{GHz}$. The simulated $A(\omega)$ peaks at $96 \%$ at 11.48 $\mathrm{GHz}$ and has a FWHM of $4 \%$ with respect to its center frequency.

By performing simulations for transmissivity and reflectivity, we are able to account for the form of the experimental data. The measured $R(\omega)$ and $T(\omega)$ are plotted as the blue curves in Fig. 3 (a) and (b) respectively. We find excellent agreement between simulated and measured $\mathrm{T}(\omega)$ over all frequencies characterized. The measured reflectance is consistently $8 \%$ lower than the simulated $R(\omega)$ at low frequencies. Both the simulated and experimental $R(\omega)$ reach a minimum at approximately $11.5 \mathrm{GHz}$, but experimentally the minimum is $11 \%$, as opposed to the simulated value of $3 \%$. There is also significant broadening in the experimental reflectivity curve with respect to simulation. This is most likely due to tolerances in the fabrication and assembly.

The experimental absorbance calculated from $T(\omega)$ and $R(\omega)$ is shown as the blue curve in Fig. 4. The simulated and experimental curves have maximum absorbance at the same frequency, $\omega_{\max }=11.5 \mathrm{GHz}$. How- 


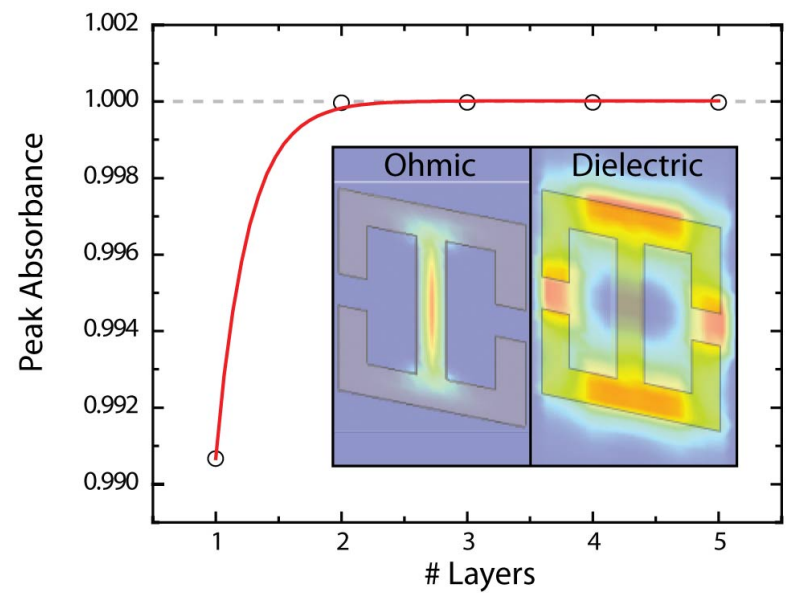

FIG. 5: Simulated absorbance with increasing metamaterial layers, red curve is a guide to the eye. Insets show the simulated losses at resonance. The Ohmic loss (surface) is shown in the left inset panel and the dielectric (volume) loss is on the right.

ever, the simulated curve reaches a maximum of $96 \%$ and the experimental curve only reaches $88 \%$. The difference between the simulated and measured curves is due primarily to deviations in $R(\omega)$. Better fabrication and assembly tolerances will permit absorbances and bandwidths similar to that shown as the red curve in Fig. 4. We performed computer simulations to verify the origin of the broadening of $\mathrm{A}(\omega)$. From a theoretical viewpoint it is expected that the frequency location of the absorbance peak depends significantly upon the separation between the electric ring resonator and the cut-wire, since this distance determines the frequency location of the magnetic resonance. Computer simulations verified this conjecture. We simulated various spacings centered about the designed value of $0.72 \mathrm{~mm}$, and computed a Gaussian weighted average $A(\omega)$ with a standard deviation of $\sigma=20 \mu \mathrm{m}$. The absorbance calculated in this manner agrees very well with the experimental curve and is plotted as the grey dashed curve in the main panel of Fig. 4. Thus assembly errors in the metamaterial spacing of only $5 \%$ can easily account for the disagreement between the simulation and experiment shown in Fig. 4 as the red and blue curves, respectively.

An investigation into the effect of adding multiple metamaterial absorbing layers is shown in the main panel of Fig. 5. As can be observed the absorbance rises sharply with additional layers and is asymptotic to unity, within computational noise. Two layers of the metamaterial absorber achieve a value of $\sim 99.9972 \%$. At this thickness the entire metamaterial is only $\sim \lambda_{0} / 2$ at resonance. For the structure shown in Fig. 1, $\mathrm{A}\left(\omega_{0}\right)$ can never reach unity since the metamaterial is not perfectly matched to free space. However, it is important to note that although an impedance matched structure may yield zero $\mathrm{R}\left(\omega_{0}\right)$, this does not necessarily mean the transmission will be small. In this study we are interested in achieving $\mathrm{R}\left(\omega_{0}\right)=\mathrm{T}\left(\omega_{0}\right)=0$ in a single unit cell in the propagation direction. Thus our metamaterial structure was optimized to maximize the absorbance with the restriction of minimizing the thickness. If this constraint is relaxed, however, an impedance matched condition can be obtained and with multiple layers a perfect absorbance can be achieved.

We further investigated the origin of the loss in our metamaterial structure, as indicated in the inset to Fig. 5. The Ohmic "surface" loss originates mainly from the center conducting region of the electric ring resonator, and dielectric losses primarily occur in-between the two metamaterial elements where the electric field is large. The major component responsible for the absorbance in our metamaterial structure is the dielectric loss and simulation indicates this is an order of magnitude greater than Ohmic losses. This is consistent with previous studies of frequency selective surfaces (FSSs) where it was found that metallic absorption was relatively insignificant in comparison to dielectric losses. 21]

We now discuss the potential application of a perfect metamaterial absorber as a bolometer. An ideal bolometric detector is one in which all photons that fall upon its surface are absorbed, converted to heat, and thus sensed.22] A prominent feature of commercially available bolometers is their extreme bandwidth making them ideal candidates as detectors, especially in the far infrared frequency regime where other efficient detection methods are largely unavailable. However, bolometers which achieve a narrowband response also have significant application as focal plane array detectors for imaging. The metamaterial design presented here achieves simulated absorbtivities near unity making them ideal candidates for bolometric pixel elements. The natural narrowband metamaterial resonance is a salient feature for focal plane array detectors as it is naturally apodizing and operates at room temperature. Since the elements which constitute our bolometer are subwavelength, metamaterials can inherently image at the diffraction limit. Further, here we have used only a single unit cell in the propagation direction, (with a thickness significantly smaller than the wavelength $\lambda_{0} / 35$ ), yet achieved an experimental absorbance of $88 \%$. By adding multiple layers one can achieve narrowband absorptivities of unity. Although the design is planar and $\mathrm{A}(\omega)$ should fall off relatively rapidly, we investigated the angular dependence (inset to Fig. (4) and found the metamaterial still achieved 50\% absorbance at an incident full-angle of $16^{\circ}$. Finally, the scalability of metamaterials permits the usage of these perfect absorbers at other wavelengths of interest such as the possibility of room temperature high resolution imaging at $\mathrm{mm}$-Wave and $\mathrm{THz}$ frequencies. This is particularly interesting owing to 
the many possible applications. We have designed similar metamaterials (not shown) as in Fig. 1 that achieved comparable $A(\omega)$ to that shown in Fig. 2 operating at $94 \mathrm{GHz}$ and $1 \mathrm{THz}$.

While the creation of a metamaterial absorber is novel, the technology to create highly absorptive materials in the microwave frequency range is well-established. The material "chiroshield" is capable of very high absorption, and reduces backscatter by 15 to $25 \mathrm{~dB}$ [23]. Chiroshield, however, has geometries on the order of one wavelength, which is 35 times larger than our design at a given center frequency. Some ferrites show reflection loss of $-30 \mathrm{~dB}$ [24]. These ferrites, however, lack the inherent and precise tunability of metamaterials. Other work has been done on the application of frequency-selective materials to bolometric devices. These bolometric designs, however, require cryogenic temperatures to operate and only achieve absorbances of about 50\% 25. At optical wavelengths, metal colloides are known to have large absorptive properties, 26] due to the geometric specific surface modes [27]. This suggests and interesting possible application of artificial plasmonic wire media[4] shaped into particular geometries to function as perfect absorbers [16] operating at microwave frequencies.

In conclusion, we have demonstrated that metamaterials can be highly absorptive over a narrow frequency range. This stems from the ability to design metamaterial elements which can individually absorb the electric and magnetic components of an incident electromagnetic wave. In contrast previous experimental results at $\mathrm{THz}$ frequencies using a single type of metamaterial element (electric) yielded values of only $A(\omega)=\sim 20 \%$. 28. The resonant frequency of the metamaterial elements may be tuned throughout some range of frequencies [29, 30] thus enabling hyperspectral imaging. A further benefit afforded by metamaterials is the ability to construct a single unit cell with $\mu(\omega)=\epsilon(\omega)$ over an extended frequency range. Thus this unit cell can achieve zero reflectance since it can have an impedance equal to the free space value $Z=\sqrt{\mu / \epsilon}=1$. This is similar to the well known theoretical construct, the Perfectly Matched Layer (PML) Absorbing Boundary Condition (ABC), 31 which splits waves incident upon a boundary into electric and magnetic components to obtain near perfect absorbtion. The PML, however, requires gain and additionally is on the order of $\lambda_{0} / 2$ in thickness 32 .

The design presented here can still be improved. By incorporating a substrate with a highly consistent dielectric constant, we will be able to optimize the design at the correct resonant frequency. Similarly, tighter fabrication tolerances would allow us to bring the impedance closer to unity and therefore closer to $100 \%$ absorption. More progress could also be made on the design itself. Currently, the absorber is polarization sensitive, which is not ideal for some applications. Additionally, the assembly is complicated due the restrictions placed on the direction of the incident wave to couple to the magnetic resonator. This resulted in variations between unit cells and less distinct absorbance peak.

NIL and WJP acknowledge support from the Los Alamos National Laboratory LDRD program, DRS acknowledges support MDA Contract W9113M-07-C-0078, and DRS, SS, and JJM acknowledge support from a Multiple University Research Initiative sponsored by the Air Force Office of Scientific Research Contract No. FA955006-1-0279.

[1] V. G. Vesalago, Sov. Phys. Usp. 10, 509 (1968).

[2] R. A. Shelby, D. Smith, and S. Schultz, Science 292, 77 (2001).

[3] D. Schurig, J. J. Mock, B. J. Justice, S. A. Cummer, J. B. Pendry, A. F. Starr, and D. R. Smith, Science 314, 977 (2006).

[4] J. B. Pendry, A. J. Holden, W. J. Stewart, and I. Youngs, Phys. Rev. Lett. 76, 4773 (1996).

[5] J. B. Pendry, A. J. Holden, D. J. Robbins, and W. J. Stewart, IEEE Trans. Microwave Theory Tech. 47, 2075 (1999).

[6] M. C. K. Wiltshire, J. B. Pendry, I. R. Young, D. J. Larkman, D. J. Gilderdale, and J. V. Hajnal, Science 291, 849 (2001).

[7] D. R. Smith, W. J. Padilla, D. C. Vier, S. C. NematNasser, and S. Schultz, Phys. Rev. Lett. 84, 4184 (2000).

[8] M. Gokkavas, K. Guven, I. Bulu, K. Aydin, R. S. Penciu, M. Kafesaki, C. M. Soukoulis, and E. Ozbay, Phys. Rev. B 73, 193103 (2006).

[9] T. J. Yen, W. J. Padilla, N. Fang, D. C. Vier, D. R. Smith, J. B. Pendry, D. N. Basov, and X. Zhang, Science 303, 1494 (2004).

[10] S. Linden, C. Enkrich, M. Wegener, J. Zhou, T. Koschny, and C. M. Soukoulis, Science 306, 1351 (2004).

[11] S. Zhang, W. Fan, N. C. Panoiu, K. J. Malloy, R. M. Osgood, and S. R. J. Brueck, Phys. Rev. Lett. 95, 137404 (2005).

[12] G. Dolling, M. Wegener, C. M. Soukoulis, and S. Linden, Opt. Lett. 32, 53 (2007).

[13] D. R. Smith and J. B. Pendry, J. Opt. Soc. Am. B 23, 391 (2006).

[14] W. J. Padilla, M. T. Aronsson, C. Highstrete, M. Lee, A. J. Taylor, and R. D. Averitt, Phys. Rev. B 75, 041102 (2007).

[15] D. Schurig, J. J. Mock, and D. R. Smith, Appl. Phys. Lett. 88, 041109 (2006).

[16] D. R. Smith, D. C. Vier, W. Padilla, S. C. Nemat-Nasser, and S. Schultz, App. Phys. Lett. 75, 1425 (1999).

[17] CST Studio Suite 2008, CST of America. http://www.cst.com (2008).

[18] D. R. Smith, S. Schultz, P. Markoš, and C. M. Soukoulis, Phys. Rev. B 65, 195104 (2002).

[19] S. G. Johnson, P. Bienstman, M. Skorobogatiy, M. Ibanescu, E. Lidorikis, and J. D. Joannopoulos, Phys. Rev. E 66, 066608 (2002).

[20] A. F. Starr, P. M. Rye, D. R. Smith, and S. NematNasser, Phys. Rev. B 70, 113102 (2004).

[21] J. E. Raynolds, B. A. Munk, J. B. Pryor, and R. J. 
Marhefka, J. Appl. Phys. 93, 5346 (2003).

[22] P. L. Richards, J. App. Phys. 76, 1 (1994).

[23] I. V. Lindell, A. H. Sihvola, S. A. Tretykov, and A. J. Viitanen, Electromagnetic waves in Chiral and Bi-Isotropic Media (Artech House, 1994).

[24] P. Singh, V. K. Babbar, A. Razdan, S. L. Srivastava, and T. C. Goel, Materials Science and Engineering B 78, 70 (2000).

[25] T. A. Perera and et al., App. Opt. 45, 7643 (2006).

[26] W. Lamb, D. M. Wood, and N. W. Ashcroft, Phys. Rev. B 21, 2248 (1980).

[27] J. B. Pendry, J. Mod. Opt. 41, 209 (1994).

[28] J. F. O'Hara, E. Smirnova, H.-T. Chen, A. J. Taylor, R. D. Averitt, M. L. C. Highstrete, and W. J. Padilla, J. Nanoelectron. Optoelectron. 2, 90 (2007).

[29] W. J. Padilla, D. R. Smith, and D. N. Basov., J. Opt. Soc. Am. B 23, 404 (2006).

[30] H.-T. Chen, J. F. O'Hara, A. K. Azad, A. J. Taylor, R. D. Averitt, D. Shrekenhamer, and W. J. Padilla, Nature Photonics - To appear (2008).

[31] J.-P. Berenger, J. Comp. Phys. 114, 185 (1994).

[32] A. Taflove and S. C. Hagness, Computational Electrodynamics: The Finite-Difference Time-Domain Method, Third Edition (Artech House, 2000). 